Using the Brain as a Metaphor to Model

Flexible Production Systems

Raghu Garud

Suresh Kotha

IS-97-36 
Using the Brain as a Metaphor to Model Flexible Production Systems

\author{
Raghu Garud \\ Leonard N. Stern School of Business \\ New York University \\ Suresh Kotha \\ Leonard N. Stern School of Business \\ New York University
}

December 1997

Working Paper Series

Stern \#IS-97-36 


\title{
USING THE BRAIN AS A METAPHOR TO MODEL FLEXIBLE PRODUCTION SYSTEMS
}

\author{
RAGHU GARUD \\ SURESH ROTHA \\ New York University
}

\begin{abstract}
Menufecturing Alexibility is critical for survivel in industries charceterized by rapid chonge and diverse product makets. Although new monufacturing technologies make it possible to accomplish flexibility, their potential remains unrealized by firms whose orgenizational olements do not possess adaptive capabilities. We use the brain as a metcphor to genercte insights on how firms might design tlexible production systems. We chose the brain as a metophor beccruse it is a self-organizing system capable of responding rapidly to a brocd renge of external stimuli. The broin as a metcphor suggests that flexibility con be enbenced by employing practices that promote distributed processes occurring in a parallel maner. Such practices lie in contrast to those employed by production systems built on scientific management principles that promote loculized processes in a sequential maner. BY exploring these contrasting modes of operation. we argue that the brain as a metcphor opens up new arenues for theory development related to the design of Elexible production systems.
\end{abstract}

Many firms compete in industries that require rapid responses to market and technological changes. Market changes reflect unpredictable customer needs for an increasing variety of products, whereas technological changes reflect continual advances that occur with the introduction of new products. In such industries, firms that possess the manufacturing flexibility to introduce modified or new products at minimal cost and lead time will goin a competitive advantage over others.

Indeed, many Japanese firms have capitalized on their monufacturing flexibility to gain worldwide competitive advontage in several industries. In contrast, it appears that U.S. manufacturing firms exhibit an astonishing lack of flexibility despite hoving invested in flexible manufacturing systems (FMS) (see Jaikumar, 1986). According to Jaikumar, it is not the technology that is to blame for this lack of flexibility, but the management of these systems (cf. Adler. 1988; Jaikumar, 1986; Pasmore, 1988; Walton \& Susmon, 1987). As a result, even though manufacturing

Authors are listed in clphabetical order. We thank Aran Kumaraswany and three amonymous reviewers for their valuable comments, and Helaine Korn. Alan Eisner, and Margaret Barry for their editorial assistance. This resecreb was supported in part by a grant from the Tenneco Fund Program at the Stern School of Business. New York University. 
Recent advances in monufacturing technologies (e.g., flexible manufacturing systems [FMS]) facilitate the manufacture of a variety of products in a continuous flow (Adler, 1988; Kotha \& Orne, 1989). However, although new flexible technologies mary be necessary, they are by no means sufficient to accomplish flexibility (Hirschhorn, 1984). This insight is best coptured by Jaikumar (1986), who asserted that U.S. manufacturers horve used flexibility the "wrong wor"-for the high-volume production of a few products. In contrast. Japmese firms have used flexibility for the production of a variety of products at lower unit costs (see also Stalk. 1988). In other words, it is not technology that is to blame for the lack of flexibility, but perhaps the management of production systems (see also Adler, 1988).

Several researchers, therefore, have suggested that firms must adopt a "systems perspective" embodying both the social and technical facets of production to achieve flexibility (e.g., Hirschhorn, 1984; Pasmore, 1988; Starbuck \& Dutton, 1973; Walton \& Susman, 1987). The time for such a broader perspective might be right beccuse new, flexible automation, relative to traditional technologies, provides a receptive framework for developing a "sociotechnical" production system (Hirschhorn, 1984). Indeed, some researchers have adopted a sociotechnical perspective to enumerate organizational factors required for attaining flexibility (e.g. Adler, 1988; Emery \& Trist, 1960; Nemetz \& Fry. 1988; Walton \& Susmon, 1987). For instance, Walton and Susman (1987) suggested changes in human resource management practices (e.g., job design, management orgorization, work-team structure, selection and training, and compensation and appraisal) to obtain the benefits of flexible cutomation. Others have argued that a system that supports learning and development is importont for attcining flexibility in production systems (Adler, 1988; Hirschborn, 1984; Nemetz \& Fry. 1988).

To operationalize these flexibility dimensions, researchers have proposed more thom 50 different flexibility types (see Chen \& Adom, 1991; Gerwin, 1993; Sethi \& Sethi, 1990; and Swamidas, 1988, for reviews). Among these, four types hare been identified as the major constructs that capture the dimensions of flexibility required in a production system: mix flexibility, volume flexibility, new product flexibility, and delivery-time flexibility (Slack, 1987; Sucrez, Cusumano, \& Fine, 1992). These four types of flexibility con be further subsumed under speed and scope flexibility (Parthasarthy \& Sethi, 1992). Speed flexibility refers to the rapidity with which a production system can deliver finished products when required, adjust its manufacturing process to the changing product mix and the accompanying volume changes, and modify its product mix. Scope flexibility refers to the breadth of products, including the degree of customization, that a production system offers.

Emerging research on flexibility suggests that speed and scope flexibility are enhanced by the ability to self-organize Jaikumar, 1986; Stalk, 1988). Self-organization permits the coordination of activities in the 
Tsoukas (1991) and Beer (1972) pointed out that the analogical link between source and target is still inconclusive becouse it is difficult to determine whether all theoretically significant aspects of the source have been captured by the torget. Hence, $a$ third level is required before conclusive comparisons between the source and target can be drawn. This is the level of "identity." This level provides a theoretical rationale for how the source and target are identical. To uncover this theoretical rationale, transformation from the level of analogy to the level of identity must preserve only core principles that describe both a source and target.

The steps discussed above generate knowledge by comparing one object in terms of another at the levels of metaphor, anclogy, and identity. This "transformational" process also is based on the possibility that what constitutes a source and $a$ target con change over time (Arbib, 1989; Gentner, 1989). An object, such as the brain, can inform our understanding of another abject, such as the computer, at one point in time. As our understonding of the computer increases over time, it can reciprocally influence our understcnding of the brain.

This transformational process need not be con isolated "top-down" movement: it can also be "oscillatory" (Tsoukas, 1991). For example, a source object $c a n$ be the basis for generating on identity from the level of the metaphor. This identity can then serve as the starting point for generating insights about the target at the level of analogy and metaphor.

We apply these steps to generate insights about flexible production systems by using the brain as $\alpha$ metaphor (see Figure 1 for details). In Step 1. we establish why the brain is an appropriate metaphor to model flexible production systems. In Step 2, we describe brain processes that render it flexible. In Step 3 (i.e., at the level of identity), we distill the "higher order" brain processes that we would then like to map to flexible production systems. From this level of identity, in Step 4, we return to the level of analogy to describe the processes that can render a production system flexible.

\section{THE BRAIN AND FLEXIBLE PRODUCTION SYSTEMS}

\section{Metcphorical Links}

As illustrated by our ability to think and converse, the human brain is capable of responding on a real-time basis to a constantly changing environment. The brain is also capable of creating new repertoires of perception and behavior as it adapts to environmental change. For example.

captured the dependency between these lower order relations in the law of gravity, the bigher order relationship. Employing the systemcticity principle, she mapped this higher order relctionship to understond the movement of electrons around a nucleus. In this wary. objects constituting the planetary system (the target) and the atom (the source) are placed in correspondence, high order relationships cre mapped (grevitational relation), and artributes of the objects constituting the source and target (such as the yellowness of the sun) are ignored. 
(1986) articulated several cybernetic principles (e.g., requisite variety, minimum critical specifications, redundancy of functions ond lecrning to learn) that organizations can apply to respond in a "brain-like fashion" to changing external stimuli.

Scholars who employ metaphors realize that a metaphorical representation is but one "constructed reality" that shapes our understanding of $\alpha$ phenomenon. We construct models of something -in this case the brain-to create $a$ model for something else-in this case the computer or an organization (Geertz, 1973). As Arbib (1989: 9) noted in his use of the brain as a metaphor to model neural networks, "A good metaphor is a rich source for hypotheses about a system, but must not be regarded as a complete theory of the system." He clso suggested the importonce of recognizing the existence of $\alpha$ two-wcy interaction between the objects being compared (Arbib, 1989: 403). With this carveat, we summarize the considerable knowledge that has been generated by artificial intelligence researchers in their efforts to mimic human intelligence through neural networks.

\section{Brain at the Analogical Level}

Many researchers suggest that the brain's ability to process information in a parallel yet distributed manner encbles it to respond rapidly to a broad range of stimuli (Anderson, 1988; Arbib, 1989; Calvin, 1990; Churchlond, 1986). The ability to process information in parallel is made possible by the brain's layered structure. Parallel processing is facilitated by firing on corroy of similar types of neurons located in modules, or knowledge areas (see Figure 2). Each neuron integrates input it receives from other neurons to generate an output. This output either excites or inhibits the activities of other neurons through synapses that establish electrochemical connections between neurons. As this process unfolds, lecming is monifested by changes in the strength of the connections between neurons in a module. Each module reaches on overall state of activity or passivity ropidly because its constituent neurons operate in parallel.

Topographical mapping is another facet of the brain that facilitates parallel processing. Topographical mapping captures interconnections between layers in the brain such that inputs from one loyer are mapped to another on $\alpha$ point-to-point basis. This process requires that $\alpha$ unique "address" for each information type exist and that information mapped to the brain be transferred through multiple channels (Calvin, 1990). ${ }^{2}$ Topographical mapping of information between the layers in the brain

\footnotetext{
2 To understand how the brein uses multiple chomnels, Calvin (1990: 148) evoled on inage of a "ribbon" cable in contrast to a single wire connecting the ignition to the starter motor in con cutomobile. The electrical connections between the ignition and the starter motor through the ribbon cable cre established by tronsmitting electrical pulses in a multichanel fashion.
} 
enhances flexibility beccuse it permits the transfer of complex information in parallel.

Research also suggests that each brain module takes part in several functions that are dispersed over several parts of the brain's anctomy. This implies that functions such as sight and sound are not localized but are distributed-i.e., several regions of the brain simultaneously participate to execute such functions. As Churchlond (1986: 162) noted, "there is a division of labor in the nervous system, but a division made many times over, a division that is fuzzy, overlapping, partially redundant, and increasingly specialized; and moreover, a division of labor that has the potential for reorgonization in the event of damage."

For distributed computation to occur, it is important to understand how various brain modules interact to perform a function. Arbib (1989) offered schemas as an intermediate construct between the brain's structure and its functioning to describe how modules interact in a distributed manner. Schemas are similar to computer algorithms in that they represent control block diagrams and flow diagrams. Block diagrams capture the feedback and feedforward loops between simultaneously active subsystems, whereas flow diagrams represent the sequencing of various data-manipulation processes.

Most systems (e.g., thermostats) have feedback and feedforward control mechanisms to ensure that performance is maintained within preset stondards. However, the brain is a system in which it is difficult to establish on a priori estimate of its operating parameters because of its continuously changing environment. In such on environment, the brain continuously updates its operating parameters that govern the transformation of information into perception and action. Arbib (1989) labeled this process tuning.

To explain how the brain tunes its operating parameters, Arbib (1989) distinguished between perceptual and motor schemas. Perceptual schemas-activated by cues from both peripheral stimuli and internal context-shape perception. Motor schemas determine an appropriate course of action. The broin tunes its operating parameters by linking perceptual schemas with motor schemas continuously to shape the operation of motor outputs. To explain this tuning process, Arbib (1989) employed an image of two layers of neurons interacting (in parallel) with one another-one being a controller and the other being a controlled surface. As neurons in the controller surface (the input perceptual schema) iterctively arrive at an overall solution, they tronsmit signals in a topographical manner to corresponding neurons in the controlled surface (the output motor schema). Churchland (1986: 446) suggested that this transfer from one surface to the other can be viewed as matrix multiplication in the brain, leading to its ability to fine tune its motor schemas even as information is received and processed by its sensory schema.

New schemas are formed by creating fresh connections between neurons within $a$ module and between modules in the brain. The repetitive 
system evolves through endogenous means involving the cooperative computation of a multitude of individual units. These units possess only local knowledge but are able to produce global solutions through their interaction. This ability to self-organize represents on identity that, when applied to cony system, con result in flexibility (Ashby, 1962; Beer, 1972; Sahal, 1979). We wish to map this self-orgonizing principle found in the brain to production systems to achieve flexibility.

Having already compared the brain with production systems at the metaphorical level, we are now left with the task of mapping insights from the brain to flexible production systems at the analogical level. Our efforts lead to several propositions. Although these propositions are consistent with emerging literature on flexibility, they offer insights that can open new arenues for exploration.

\section{Production Systems at the Level of Analogy}

The notion of cooperative computation as applied to flexible production systems implies distributed processing of activities occurring in parallel. We argue that distributed processing enhonces the scope flexibility of production systems, whereas parallel processing enhances their speed flexibility. We establish these arguments by comparing flexible production systems with traditional production systems that were designed with scientific management principles (see Table 1 for a comparison). Specialization through division of labor and standardization and hierarchy are key building blocks of scientific management. Specialization results in localized processing of activities; hierachy results in sequential processing. In the following sections, we explain how these processes result in compromising scope and speed flexibility.

Traditional production systems are designed to deal with environmental change by buffering their production cores with inventories (Thompson, 1967). These inventory buffers decouple production systems from their environments, thereby creating production systems that are "islands into themselves" (Hackman, 1990). In such a system, customer . demands are serviced by drawing upon an inventory of finished goods. This mode of operation, labeled as "speculation" (Stern \& El-Ansery, 1982), commits the system to a predetermined course of action based on a forecast of the future. Production is undertaken to stock inventories by engaging the services of highly specialized functional areas (e.g., design, engineering, manufacturing) in a sequential manner coordinated through a hierarchy. In contrast, flexible production systems must possess the ability to "postpone" the creation of products until customer requirements are known. At the extreme, production commences only ofter the production system senses stimuli originating from customers, i.e., just in time.

Topographical mapping is a key process in the brain that enhances its ability to sense and respond to external stimuli just in time. Topographical mapping in the brain requires that there exist a unique address for each type of stimulus and that information be mapped on a 
versity of product attributes sought by customers. This allows a one-toone mopping of customer attributes to corresponding value centers ${ }^{3}$ in the production system. This discussion leads to the following proposition:

Proposition 1: Production systems that undertake isomorphic mapping will exhibit greater scope flexibility relative to those that undertake homomorphic mapping.

The second facet of topographical mapping (i.e., multi-chonnel processing) enables the brain to process information in porallel, thereby enhancing the ropidity with which it can respond to stimuli. Similarly, the extent to which a production system also employs multi-channel mapping enhances its speed flexibility. Multi-chonnel mapping in a production system is a process by which information about customer needs is transmitted in parallel to different value centers. For instance, a product with two attributes - functionality and cost-must evoke responses in the design and manufacturing departments, respectively.

von Hippel (1994) offered the notion of "sticky data" to explain why production systems must employ multi-channel mapping to accomplish speed flexibility. Data are sticky when there are costs associated with replicating and diffusing "location-specific" information. "He argued that sticky data con hare $\alpha$ significant impact on the locus of problem solving. sometimes requiring that problem-solving activity shift to the location where sticky datc reside. He clso suggested partitioning problems so that subparts of the problem are directed to specific sites where appropriate sticky data reside.

As in the brain, this approach requires multi-channel processing in which information is directed to specific locations in the production system. Directing specific parts of a problem to appropriate locations through a multi-channel transfer process obviates the need for transferring location-specific information from one place to mother. As a consequence, the rapidity and accuracy of responses increase.

${ }^{3}$ A value center represents $a$ collection of similar activities undertaken by humans and machines to convert basic row materials to finished goods. The isomorphie mapping process is illustrated by Quality Function Deployment (QFD). QFD is a mapping technique for the development of new products through interfunctional planning and communication (Houser \& Clausing, 1988). This mapping process is illustrated by considering a grid in which one aris details customer expectations und the other represents every conceivable product characteristic. At the points on the grid where the vertical and horizontal ares intersect, the developers assign a degree of correlation between the market need ond the product characteristics. The completed chat provides guidelines that designers and others can use to develop the most appropricte product.

- Sticky datc emerge beccuse of encoding, coupling, and diffusion costs. Encoding costs arise becouse of difficulties associated with embedding and recontextualizing transferred lowowledge at the receiving site. Coupling costs crise because of difficulties associated writh integrating tronsferred knowledge with complementary knowledge at the receiving site. Diffusion costs arise because of difficulties associated with transferring datc from one site to cmother. 


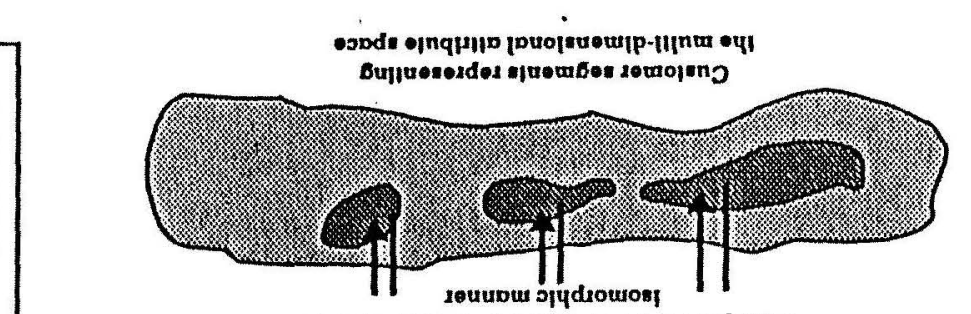

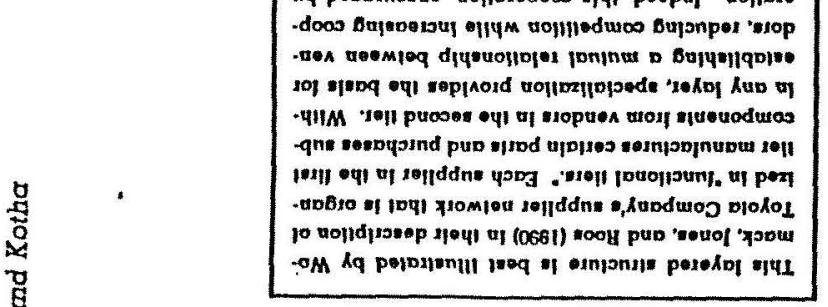

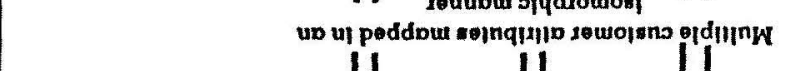

megues enjpa Buourd pur use M10q suop|soutos

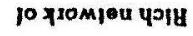
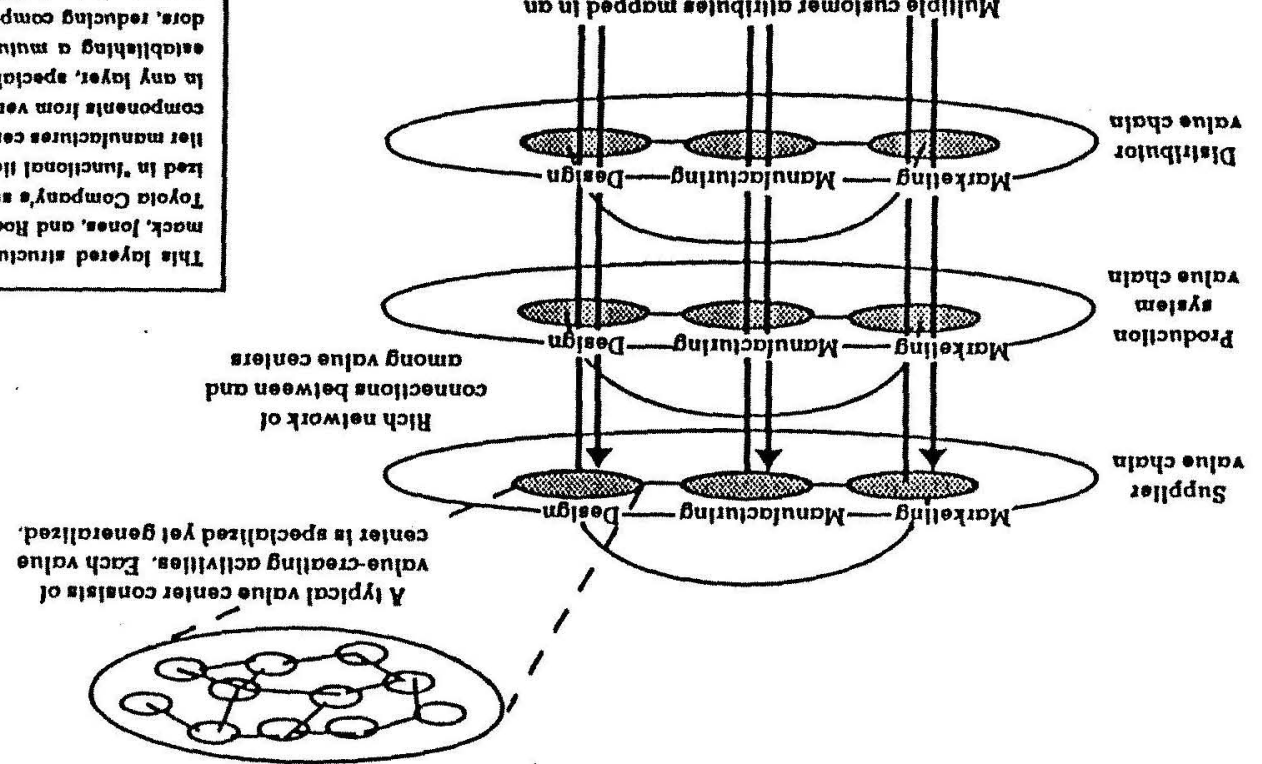

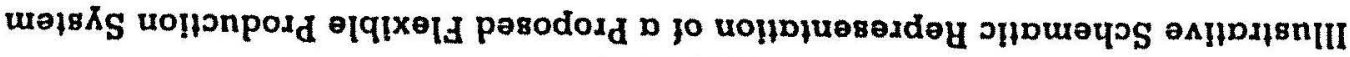


weakening others, rather than creating completely new ties while dissolving others.

As Eccles and Crane (1988) suggested, the dynamic network structure functions by establishing "strong" and "weak" ties between various value centers that transcend firm boundaries. Strong ties, formed between centers that interact frequently, are essential for carrying out day-to-day production activities. ${ }^{5}$ Weak ties are important to establish what Hirschhorn (1984: 92) called "fringe cworeness." Under changing conditions, the continuous feedback received by a system at the fringe of awareness, along with conscious planning at the "center of awareness," enables $\alpha$ system to respond flexibly.

In contrast to the process described above, traditional production systems hove been designed as hiercrchical structures in which value centers are selectively linked with others (representing $\alpha$ chein of command), and each value center is, at best, sequentially interdependent with activities undertaken by other centers of the hierarchical system. Such $\alpha$ hierarchical structure creates rigidities becoruse system evolution is governed through time-consuming exogenous processes. This discussion is the basis for the following proposition:

Proposition 3: Production systems orgcrized as a dynamic network will exhibit greater speed and scope flexibility relative to those that are organized as $\alpha$ bierarchy.

The brain is able to create a dynamic network through distributed processing beccuse each brain module con undertake functions performed by other modules. Thus, even though modules are specialized. they have generalized capabilities; that is, the division of labor in the broin is fuzzy, overlapping, and portially redundont (Churchlond, 1986). Similarly, distributed computation in production systems will be facilitated if value centers possess generalized capabilities despite being specialized in porticular tasks. This is what Imai, Nonaka, and Takeuchi (1985) labeled as "shared" division of labor.

Shared division of labor facilitates speed flexibility in several ways. First, beccuse of its generalized competencies, each center can operate semi-cutonomously, keeping the requirements of other centers as

\footnotetext{
${ }^{3}$ Granovetter (1973) bas shown that centers that exhibit strong ties can become isolated trom other sources of information unless they also create weak ties with others outside their own group. Weak ties - those that arise through occasional contacts-cre con important way of linking centers with strong ties with other centers, thereby crvoiding merging them into $a$ larger group of strong ties (see also Eccles \& Crone, 1988: 132).

${ }^{6}$ Multi-skilling is one menifestetion of shered division of labor. It implies the acquisition of skills that cut across functions. In environments in which product rariety and speed require fluid responses, multi-skilling overcomes the rigidities that set in from the division of labor (Adler, 1988; Eccles \& Crane, 1988; Hares "\& Wheelwright. 1988; Pasmore. 1988, Walton \& Susmen. 1987).
} 
speed flexibility during manufacturing as different value centers are unable to work in parallel to create modules that can be assembled just in time. This discussion is the basis for the following proposition:

Proposition 5: Production systems that employ modularized product designs will exhibit greater speed and scope flexibility relative to those that employ integrated product designs.

How can a production system rapidly and reliably sense the mix of product attributes required to fulfill customer needs? The cooperative computation style of the brain suggests that the process should evolve through the cooperative interactions of different value centers. In this process, no predetermined plans bind value centers to a particular course of action. The cooperative computation style exploits information as it becomes arcilable at each value center. Based on inputs received through the multi-chomel mapping process, each value center formulates tentative hypotheses about the specific attributes required by customers. Information thus received forms the basis for a set of generic activities that each value center undertakes. These generic activities provide the foundation for future refinements that the system con undertake when more information becomes available. ${ }^{7}$ For example, the Benetton Compony, sensing the demand for sweaters, initiates activities for the creation of "generic" (plain light-gray color) sweaters and postpones the dying process until exact fashion trends become clecrer.

Mediated by organizational routines, each value center can begin tuning its activities to arrive at an overall configuration of product attributes. This is an iterative process whereby each center constrains other centers and, in turn, is constrained by them. As different value centers evolve to an overall understanding of the mix of product attributes required by customers, they are able continuously to update operating parameters that govern their conversion processes.

Sahal (1979) labeled the ability to update operating parameters to cater to environmental shifts as "homeorhesis." Homeorhesis represents the capacity of a system not merely to return to its state before the occurrence of a disturbance, but to seek new development pathwarys through

7 This form of cooperative computation is illustrated by examining the overlopping progrom phases employed by many Japanese manufacturing firms during the new productdevelopment process (see, for example, Imai ot al., 1985). Historically, new productdevelopment efforts or activities were carried out in sequence with different parts of a flexible production system. such as marketing. design. engineering, and manufacturing. sequentially actucted over time. In contrast, con overlopping product-development process requires the involvement of all the functions at the early stages of new product development. Even though some furctions may be underutilized at different periods during the productdevelopment process. the overlop between the various phases and consequent parallel yet distributed processing of information speeds up the development and introduction of new products. 
Similarly, organizational routines, too. can create blind spots (Henderson \& Clark, 1990) and commit an organization to a course of action that is not in tune with its environment (Allison, 1972). Moreover, as captured in the notion of absorptive capacity (Cohen \& Levinthal, 1990), the existing stock of competencies affects an organization's ability to perceive, assimilate, and act upon data. The powerful influence of routines in shaping perception and committing organizations to a mode of operation is illustrated by the delay of mony U.S. firms in shifting to "just-intime" routines despite evidence of its superiority over "just-in-case" routines.

A brain preserves its vitality despite the potential for blind spots and rigidities by assimilating new knowledge and creating new schemas. Over time, $\alpha$ brain develops a broad knowledge base and $\alpha$ repertoire of schemas. The wider the knowledge base and the repertoire of schemas, the greater its ability to recognize and respond to a wide variety of stimuli. Similarly, flexible production systems must possess a repertoire of organizational routines and competencies to overcome potential blind spots and rigidities. Building upon the notion of absorptive capacity, we suggest that the broader the existing base of competencies and routines, the greater the production system's ability to perceive and assimilate extemal stimuli. A broad base of competencies and routines also enhances the production system's ability to respond to a wide range of customer demonds (Ashby, 1965). Therefore, these capabilities enhonce scope flexibility.

- Moreover, as in the broin, $\alpha$ production system's ability to preserve its vitality is contingent upon whether it continuously updates its competencies and routines (Hoyes \& Wheelwright, 1988; Pasmore, 1988). One way to accomplish this is to institute major reorganizations periodically (Eccles \& Crane, 1988; Hommer, 1990). Reorganization provides on opportunity for a firm to seek new developmental pathways; $\alpha$ procedure that Eccles and Crane (1988: 143) likened to the "connealing process used in crystal formation." In this way, periodic reorganization helps overcome rigidities that might otherwise set in. This discussion is the basis for the following proposition:

Proposition 7: Production systems with a broad base of competencies and routines that are continuously updated will exhibit greater scope flexibility relative to those that possess $\alpha$ narrow base of competencies and routines that are updated intermittently.

The value of the metaphorical approach that we have adopted lies in our ability to generate insights that can be mapped from a target to a source. These insights, as transferred from the brain to flexible production systems, are summarized in Table 2. However, the utility of the insights gained from $\alpha$ metaphor, and the boundary conditions over which these insights are valid, must be empirically established. Toward this 
tem produces (e.g. Jaikumar, 1986; Tombak, 1988) and assessing its ability to deliver customized products (Parthasarthy \& Sethi, 1992).

Multi-chomel processing might be evidenced by the presence of multiple points of contact between the different layers of a production system (i.e., customers, distributors, suppliers, and manufacturers). Multichomnel processing also requires that researchers excmine the degree to which information between the layers is processed in a parallel manner.

Whether a production system is capable of isomorphic mapping can be gouged by assessing the range of competencies it possesses relative to the diversity of product attributes required by customers. The gap between what is required by customers and what the production system can create provides $\alpha$ measure of the system's capability to map isomorphically. Assessing whether a production system employs techniques such as the "quality function deployment technique" (Hauser \& Clausing, 1988) is also indicative of its ability to map isomorphically.

Dynamic network properties may be assessed by a system's ability to create temporary teams and the extent to which lateral, rather thon hierarchical, integration mechomisms are employed. Measures such as the degree of formalization, delegation, specialization, and integration that place firms along a continuum between organic and mechmistic organizations (e.g., Decm, Yoon, \& Susman, 1992; Von de Ven \& Ferry, 1980) and integrative mechanisms (e.g., task teams) proposed by Galbraith (1973) may be employed with appropriate modifications. The extent to which information processing is centralized or decentralized can also be assessed.

Researchers can operationalize the notion of modularity by using the dimensions of modularity described by Pine (1993) and Wheelwright and Clark (1992). The degree to which component redundancies are used for mixing and matching components to achieve product variety and the extent to which standardized interface specifications are employed can also be used to operationalize this construct.

Resecrchers may gouge the emphasis placed on shared division of labor at three levels - the individual, group, and production system. At the individual level, resecrrchers moy focus on the degree to which employees are multi-skilled, the extent to which machines and equipment possess capabilities that go beyond those required to perform one task, and the extent to which job rotation is practiced within the production system. At the group level, the employment of redundaricies in new product-development teams moy be assessed. At the production system level, the concept can be gauged by evaluating the extent to which firms possess competencies in which others (i.e., suppliers) might speciclize.

Tuning is a measure of the rapidity and ease with which $\propto$ production system can change its production parameters to accommodate product mix changes. The application of concurrent engineering principles via the use of CAD and CAM mary provide an indication of a production system's ability to tune its production parameters on $a$ real-time basis. 
To the extent designers embrace $\alpha$ machine model for the production of flexible production team[st. they are unlikely to reap the very benefits to which they aspire-benefits that well-designed and well-led work teams are in fact capable of providing.

Here we con see why investing in technology alone will not lead to flexibility. The scientific management paradigm is fundamentally incompatible with efforts to design flexible production systems (see Hackmon, 1990; Hirschhom. 1984). Discovering the "one best wary" to accomplish tasks through specialization results in fragmentation rather than synthesis, and therefore, is antithetical to self-organization through teamwork (Hackman, 1990). Making incremental changes within this paradigm is unlikely to lead to new insights about manufacturing flexibility. U.S. manufacturing firms need a new paradigm that can generate novel insights on how to accomplish flexibility.

We offered the brain as a metaphor to create such a new paradigm. Brain processes that enhonce its ability to respond rapidly to a broad range of stimuli lie in contrast to localized and sequential processing found in traditional production systems. Specifically, brain processes unfold in a parallel yet distributed manner through a cooperative computation process. Cooperative computation enhances self-organization capabilities, the key to realizing flexibility in ony system.

These observations have important implications for theory and practice that must be directed at identifying how U.S. manufacturing firms can carry out value creation in a parallel yet distributed manner. Production systems must be designed to abandon hierarchical modes of command and control to facilitate parallel processing. Production systems must also be designed to break down barriers that isolate functional activities so that value-creating centers can be combined in novel warys to address customer needs, thereby facilitating distributed processing. Most importantly, in the image of the brain, production systems must be designed to foster a learaing environment in which the system is able to evolve incrementally from initial inputs.

We have tapped only a small fraction of the insights that the brain as a metaphor has to offer. Existing knowledge about the brain provides $\alpha$ rich theoretical base for generating new insights on flexibility. Equally important, researchers continue making new discoveries obout the brain that can provide additional insights on flexibility. Thus, using the brain as a metaphor to model flexible production systems not only provides us with a knowledge base to work from, but a knowledge base to work with.

\section{REFERENCES}

Adler. P. S. 1988. Managing Ilexible automation. Califomia Management Review, 20(1): 35-56.

Allison. G. T. 1971. Essence of decision. Boston: Little, Brown and Company. 
Hauser. J. R., \& Clausing. D. 1988. The house of quality. Harrard Business Review, 66(3): $63-73$.

Hcyes, R. H., \& Wheelwright, S. C. 1979. The dypamics of process-product life cycles. Harrard Business Review, 57(2): 15-22.

Hayes, R. H., Wheelwright. S. C., \& Clark. K. B. 1988. Dracmic manufacturing. New York: Free Press.

Henderson. R. M.. \& Clark, K. B. 1990. Architectural innovation: The reconfiguration of existing product technologies and the failure of established firms. Administratire Science Quarterly, 35: 9-30.

Hirschhorn. L. 1984. Beyond mechanization: Work and technology in a postindustrial age. Cambridge, MA: MIT Press.

Imai. K. Nonaka. I., \& Takeucbi. H. 1985. Managing the new product development process: How Japonese componies lean and unlean. In K. B. Clark. R. Hayes, \& C. Lorenz (Eds.). The uneasy alliance: Managing the productivity technology dilemma: 337-375. Cambridge, MA: Harvard Business School Press.

Jaikumar. R. 1986. Postindustrial manufacturing. Harvard Business Review, 64(6): 69-76.

Kotha. S.. \& Orne, D. 1989. Generic manufacturing strategies: A conceptual synthesis. Strategic Management lournal. 10: 211-231.

Morgan. G. 1986. Images of organization. Beverly Hills, CA: Sage.

Nemetz. P. L.. \& FrY. L. W. 1988. Flexible manufacturing orgemizations: Implications for strategy and organization design. Academy of Management Review, 13: 627-638.

Nonaka. I. 1991. Managing the firm as an information creation process. In I. Meindl (Ed.). Adrances in information processing in organizations, vol. 4: 239-275. Greenwich, CT: JAI Press.

Parthasarthy, R., \& Sethi. S. P. 1992. The impact of flexible automation on business strategy and organizational structure. Academy of Mancgement Review. 17: 86-111.

Pasmore. W. A. 1988. Designing effective organizations: The sociotechnical systems perspective. New York: Wiley.

Pine II. B. J. 1993. Mass customization. Boston: Harvard Business School Press.

Schal, D. 1979. A unified theory of self-orgmization. Jound of Cybernetics, 9: 127-142.

Sethi. A. K. \& Sethi. S. P. 1990 . Flexibility in manufacturing: A survey. The lateractional Journal of Flexible Manufacturing System. 2: 289-328.

Slack, N. 1987. The flexibility of manufacturing systems. International Journal of Operations and Production Monagement, 7(4): 35-45.

Stalk, G. 1988. Time: The next source of competitive advontage. Harvard Business Review, 66(4): $41-51$.

Starbuck, W. H., \& Dutton. J. M. 1973. Designing adaptive organizations. Journal of Business Policy. 3(4): 21-28.

Stern. N., \& El-Ansery, A. I. 1982. Marketing channels. Englewood Cliffs, N]: Prentice Hall.

Sucrez. F. F., Cusumano. M. A.. \& Fine, C. H. 1992. Flexibility and performance: A literature critique and strategic framework. Working poper. Sloon School of Monagement, Massachusetts Institute of Technology. Combridge.

Swamidass, P. M. 1988. Manufacturing flexibility. Operations Management Association, Monograph No. 2.

Toylor. F. W. 1967. Principles of scientifie management. New York: Norton.

Thompson. J. D. 1967. Organizertions in cetion. New York: McGraw-Hill.

Tombak. M. 1988. The importance of flexibility in manufacturing. Working paper, 88/33. INSEAD. Fontcinblecru. Frence. 